Article

\title{
Investigation of the Microstructures of Graphene Quantum Dots (GQDs) by Surface-Enhanced Raman Spectroscopy
}

\author{
Junxiao Wu, Peijie Wang, Fuhe Wang and Yan Fang * \\ The Beijing Key Laboratory for Nano-photonics and Nano-structure, Department of Physics, Capital Normal \\ University, Beijing 100048, China; wujxcnu@163.com (J.W.); 2140602054@cnu.edu.cn (P.W.); \\ wangfhcnu@126.com (F.W.) \\ * Correspondence: fangyan@cnu.edu.cn; Tel.: +010-6890-3565
}

Received: 21 September 2018; Accepted: 19 October 2018; Published: 22 October 2018

\begin{abstract}
Photoluminescence (PL) is the most significant feature of graphene quantum dots (GQDs). However, the PL mechanism in GQDs has been debated due to the fact that the microstructures, such as edge and in-plane defects that are critical for PL emission, have not been convincingly identified due to the lack of effective detection methods. Conventional measures such as high-resolution transmission electron microscopy and infrared spectroscopy only show some localized lattice fringes of GQDs and the structures of some substituents, which have little significance in terms of thoroughly understanding the PL effect. Here, surface-enhanced Raman spectroscopy (SERS) was introduced as a highly sensitive surface technique to study the microstructures of GQDs. Pure GQDs were prepared by laser ablating and cutting highly oriented pyrolytic graphite (HOPG) parallel to the graphite layers. Consequently, abundant SERS signals of the GQDs were obtained on an Ag electrode in an electrochemical environment for the first time. The results convincingly and experimentally characterized the typical and detailed features of GQDs, such as the crystallinity of $\mathrm{sp}^{2}$ hexagons, the quantum confinement effect, various defects on the edges, $\mathrm{sp}^{3}$-like defects and disorders on the basal planes, and passivated structures on the periphery and surface of the GQDs. This work demonstrates that SERS is thus by far the most effective technique for probing the microstructures of GQDs.
\end{abstract}

Keywords: graphene quantum dots; surface-enhanced Raman spectroscopy; photoluminescence; laser ablation; passivation

\section{Introduction}

Graphene quantum dots (GQDs), as a new type of zero-dimensional quantum dot, have drawn intense attention due to their unique properties, such as chemical stability, low toxicity, dispersibility in water, controllable size, and wavelength tenability [1-5]. GQDs have great potential for applications in white light-emitting devices [6,7], fluorescent probes [8,9], anticancer therapy [10], biosensors and photocatalysis [11-13]. Nano-sized GQDs are composed of $\mathrm{sp}^{2}$ hexagonal domains and functional groups around the periphery and surface of the GQD domains. The GQD domains have a band gap that can be tuned by changing their size and shape [14-16]. The periphery and surface of the GQD domains can be chemically passivated by electron-donating substituents. Consequently, GQDs exhibit photoluminescence (PL) due to the quantum confinement effect and surface passivation effect [17-22].

Thus far, the PL mechanism in GQDs has been debated and therefore requires further clarification. Photoluminescence excitation (PLE) spectra of the GQDs generally show two PL peaks, indicating the two transitions from the triplet energy states of GQDs $[17,19,23]$. Based on the triplet ground-state 
carbene model, these two transitions could be considered to be from $\sigma$ and $\pi$ to $\pi^{*}$ due to the electronic conjugate structures, free zigzag sites, and in-plane defects of the GQD domains [19,23]. Another model suggests that the surface state energy levels could be created between $\pi$ and $\pi^{*}$ states due to the functional groups on the periphery and surface of the GQDs, resulting in two transitions from $\pi$ and surface states to the $\pi^{*}$ state, respectively [17]. Therefore, the PL peaks should be determined by the size, structure and defects of the GQD domains, such as $\mathrm{sp}^{2}$ hexagons, edge and in-plane defects, and the chemically passivated structures on the periphery and surface of GQDs. The passivated structures of electron-donating substituents seem to have been partly characterized by Fourier transform infrared (FTIR) spectroscopy, X-ray photoelectron spectroscopy (XPS), etc. [17]. However, the distinct structures of GQD domains, especially the structures of edge and in-plane defects that are always formed and incorporated in $\mathrm{sp}^{2}$ hexagons in the synthesis process, have not been reported due to the lack of an effective structure detection method. Only some localized lattice fringes and obscure edge structures of GQD domains, rather than the detailed structures of the defects, were observed by transmission electron microscopy (TEM) and high-resolution transmission electron microscopy (HRTEM) [24,25]. Although the structures of edge and in-plane defects in GQDs have been theoretically presumed to significantly affect the photoluminescence of GQDs [26], little experimental evidence related to the fine structure of defects has been presented, leading to ambiguity.

GQDs have usually been fabricated via top-down cutting routes and bottom-up synthesis methods [27], such as hydrothermal methods [5,28-30], enhanced hydrothermal methods [31,32], carbonization [23], acidic exfoliation routes [33], and electrochemical strategies [34,35]. These methods suffer from complex and severe formation processes, including high temperature and concentrated acid environments $[33,36,37]$, inevitably leading to the existence of chemical additives, residues and stabilizers. These impurities in the GQD samples not only complicate the understanding and theoretical simulations of the GQD PL mechanism via the GQD microstructures, but also affect the Raman and surface-enhanced Raman spectroscopy (SERS) measurements of the GQD fine structures, in which pure GQD samples are needed.

Raman spectroscopy is a powerful tool in the structural characterization of graphitic materials [38,39]. However, the Raman spectra of GQDs usually only show a D band at approximately $1350 \mathrm{~cm}^{-1}$ and a G band at approximately $1588 \mathrm{~cm}^{-1}[19,40-43]$. There is a lack of more Raman bands associated with the structure of GQDs. Surface-enhanced Raman spectroscopy (SERS), as a highly sensitive surface technique, can greatly enhance the Raman signals of the molecules adsorbed on noble metal nanoparticles [44-46], offering the chance to detect more Raman bands corresponding to the detailed structure of the GQDs.

In this paper, pure GQDs without chemical additives, residues and stabilizers were fabricated for the SERS study by ablating highly-oriented pyrolytic graphite (HOPG) with a pulsed laser beam along the orientation parallel to the graphite layers in deionized water. The SERS spectra of GQDs with abundant peaks were obtained by the chemisorption of GQDs on Ag electrodes in an electrochemical environment. In contrast to the case of two-dimensional large graphene sheets, the D band split into two peaks at $1362 \mathrm{~cm}^{-1}$ and $1387 \mathrm{~cm}^{-1}$ due to the double resonance effect of graphene. The lower frequency peak related to the edge defects was stronger than the higher frequency peak that was derived from disorders such as $\mathrm{sp}^{3}$ structures and the fluctuation of the curvature on the basal planes. The D band was further broadened and blue shifted due to the various defects, disorders and passivated functional groups of the GQDs. The G band was also split into two peaks by symmetry breaking. The additional phonon modes demonstrated that the basal planes of the GQD domains retained the $\mathrm{sp}^{2}$ hexagonal structure. The abundance of microstructures in the SERS spectra that were not directly observed with other conventional detection and observation methods indicated that the microstructures of the GQDs could be effectively characterized by SERS. The results contributed to the further understanding of the types of defects and their effects on the PL mechanism in GQDs. 


\section{Materials and Methods}

Silver nanoparticle suspensions were prepared by ablating a silver plate in deionized water. The average diameter of the silver nanoparticles was approximately $30 \mathrm{~nm}$. The silver plate was then replaced by highly oriented pyrolytic graphite (HOPG, SPI Supplies Co., West Chester, PA, USA) to fabricate the GQD samples via the ablation of the HOPG along the orientation parallel to the graphite layers in deionized water. Ablation was achieved with a Q-switched Nd: YAG laser with a wavelength of $1064 \mathrm{~nm}$, a repetition rate of $10 \mathrm{~Hz}$, and a pulse width of $6 \mathrm{~ns}$. Consequently, GQDs adequately combined with the silver nanoparticles to form the Ag@GQD samples.

The Ag@GQDs sample was coated on a roughened silver electrode (99.9\%) that was placed in a typical electrochemical cell containing a $0.1 \mathrm{M} \mathrm{Na}_{2} \mathrm{SO}_{4}$ solution. A platinum wire and an $\mathrm{Ag} / \mathrm{AgCl}$ electrode were employed as the counter electrode and reference electrode, respectively. The voltage applied to the working electrode was controlled by a $\mathrm{CHI} 660 \mathrm{~A}$ electrochemical instrument $(\mathrm{CH}$ Instrument, Bee Cave, TX, USA) The SERS spectra of GQDs on the surface of the working electrode were collected with varying potentials from $0.0 \mathrm{~V}$ to $-1.2 \mathrm{~V}$.

The morphology of the GQDs was observed by TEM and HRTEM (Tecnai G2 F30, FEI Company, Hillsboro, OR, USA) with an acceleration voltage of $300 \mathrm{kV}$, accompanied by energy disperse spectroscopy (EDS) measurements. The PL emission spectra were measured with a fluorescent spectrophotometer (F-7000, Hitachi, Tokyo, Japan). The Raman and SERS spectra were measured with a confocal micro-Raman spectrophotometer (Renishaw H 13325, Renishaw PLC, London, UK) and excited at the wavelength of $532 \mathrm{~nm}$. The power of the laser used on the sample was $0.41 \mathrm{~mW}$. Infrared (IR) absorption spectra were recorded with an FTIR spectrometer (60 SXB, Nicolet Instrument. Inc., Madison, WI, USA) using a $\mathrm{KBr}$ wafer with a resolution of $4 \mathrm{~cm}^{-1}$. XPS spectra were collected using an ESCALAB 250 X-ray photoelectron spectrometer (Thermo Fisher Scientific Inc., Waltham, MA, USA) with a spatial resolution less than $3 \mu \mathrm{m}$.

\section{Results and Discussion}

Figure 1a shows TEM images of GQDs fabricated by ablating HOPG with a pulsed laser beam along the orientation parallel to the graphite layers. GQDs with diameters of 2-6 nm were uniformly and densely dispersed in the products. The HRTEM images show localized lattice fringes of graphene with an in-plane lattice parameter of approximately $0.24 \mathrm{~nm}$ (Figure 1b) that can be ascribed to (1120) [23]. The (002) lattice spacing distance was $0.35 \mathrm{~nm}$ (Figure 1c), slightly larger than that in bulk graphite $(0.336 \mathrm{~nm})$ [19]. The PL emission of GQDs excited at a wavelength of $400 \mathrm{~nm}$ showed a peak centered at $473 \mathrm{~nm}$ without chemical passivation additives (inset of Figure 1a). Notably, when the HOPG was ablated in the direction perpendicular to the graphite layers, the samples mainly included carbon nanoparticles similar to those that are conventionally achieved when ablating candle soot and graphite powder with a laser [24,47].

Figure 2 shows the XPS, FTIR and EDS spectra of the GQDs. Overall, these measurements represent an attempt to visualize the structure of the GQDs.

The XPS spectrum shows sharp peaks at binding energies of $285 \mathrm{eV}(\mathrm{C} 1 \mathrm{~s})$ and $532 \mathrm{eV}(\mathrm{O} 1 \mathrm{~s})$ (Figure 2a). The high-resolution spectrum of the C1s (Figure 2b) shows a wide band consisting of three components at 284.8, 286.5 and $288.7 \mathrm{eV}$, which are attributed to graphitic, hydroxyl, and carboxylic groups on the periphery and surface of the GQDs, respectively $[17,23]$. The proportion of the graphitic peak suggests that carbon was the main component of the GQDs.

In the FTIR spectrum (Figure 2c), two typical peaks at 3434 and $1384 \mathrm{~cm}^{-1}$ were observed and could be assigned to the stretching vibration and in-plane bending vibration of $\mathrm{C}-\mathrm{OH}$, respectively. An intense peak at $1632 \mathrm{~cm}^{-1}$ was ascribed to the vibration of $\mathrm{C}=\mathrm{C}$. In addition, the absorption of $\mathrm{C}-\mathrm{O}$ in the carboxylate groups at 1039 and $1134 \mathrm{~cm}^{-1}$ and the weak stretching vibration of $\mathrm{C}-\mathrm{H}$ at $2960 \mathrm{~cm}^{-1}$ were observed [17,23]. The IR characterization of GQDs was distinctly different from that of the HOPG [48]. 
The EDS spectrum shows that the GQDs contain carbon and oxygen (Figure 2d). The atomic ratio of $\mathrm{C} / \mathrm{O}$ was approximately $9 / 1$, indicating that $\mathrm{C}$ was the dominant element in the GQDs.

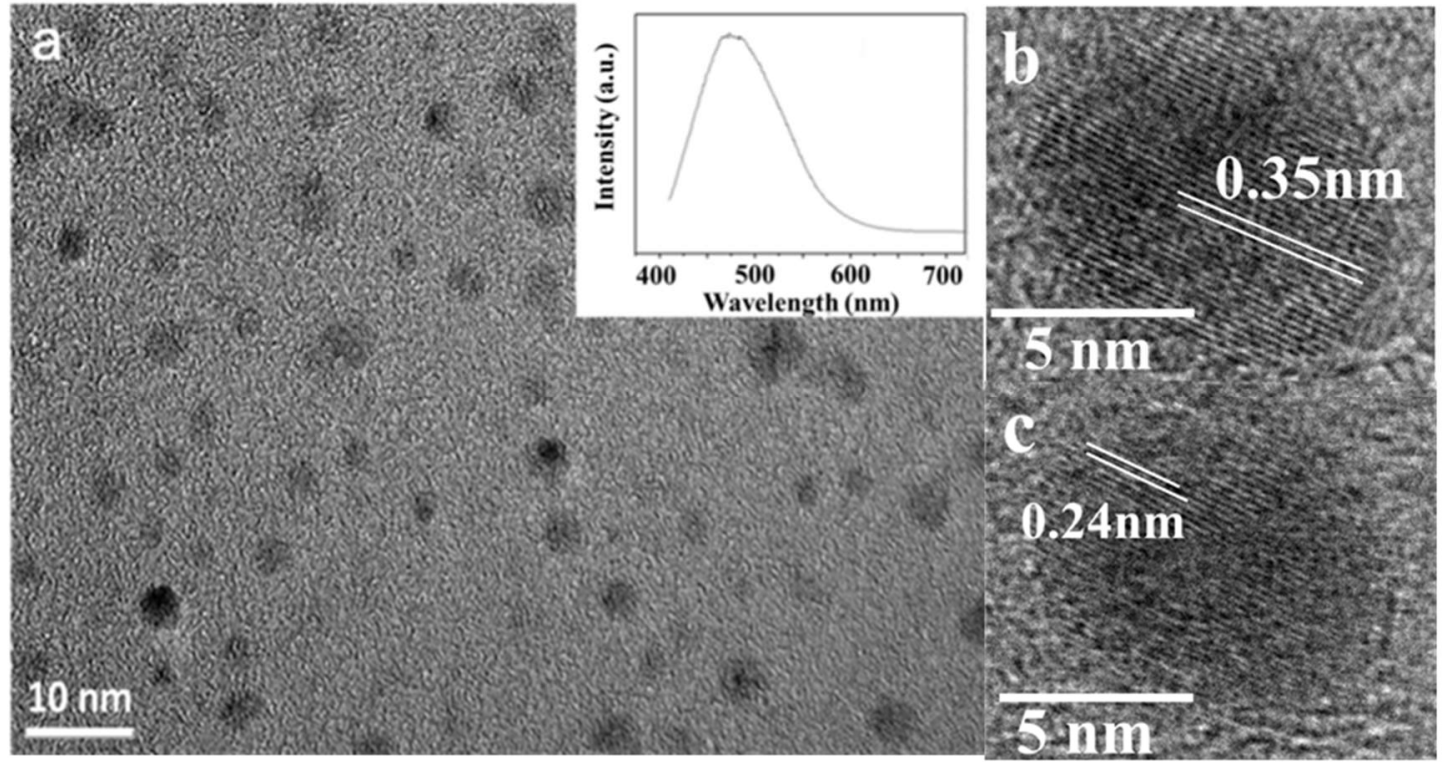

Figure 1. (a) TEM images of graphene quantum dots (GQDs) and the photoluminescence (PL) spectrum of GQDs excited at a wavelength of $400 \mathrm{~nm}$ (inset); (b) and (c) High-resolution transmission electron microscopy (HRTEM) images of GQDs.
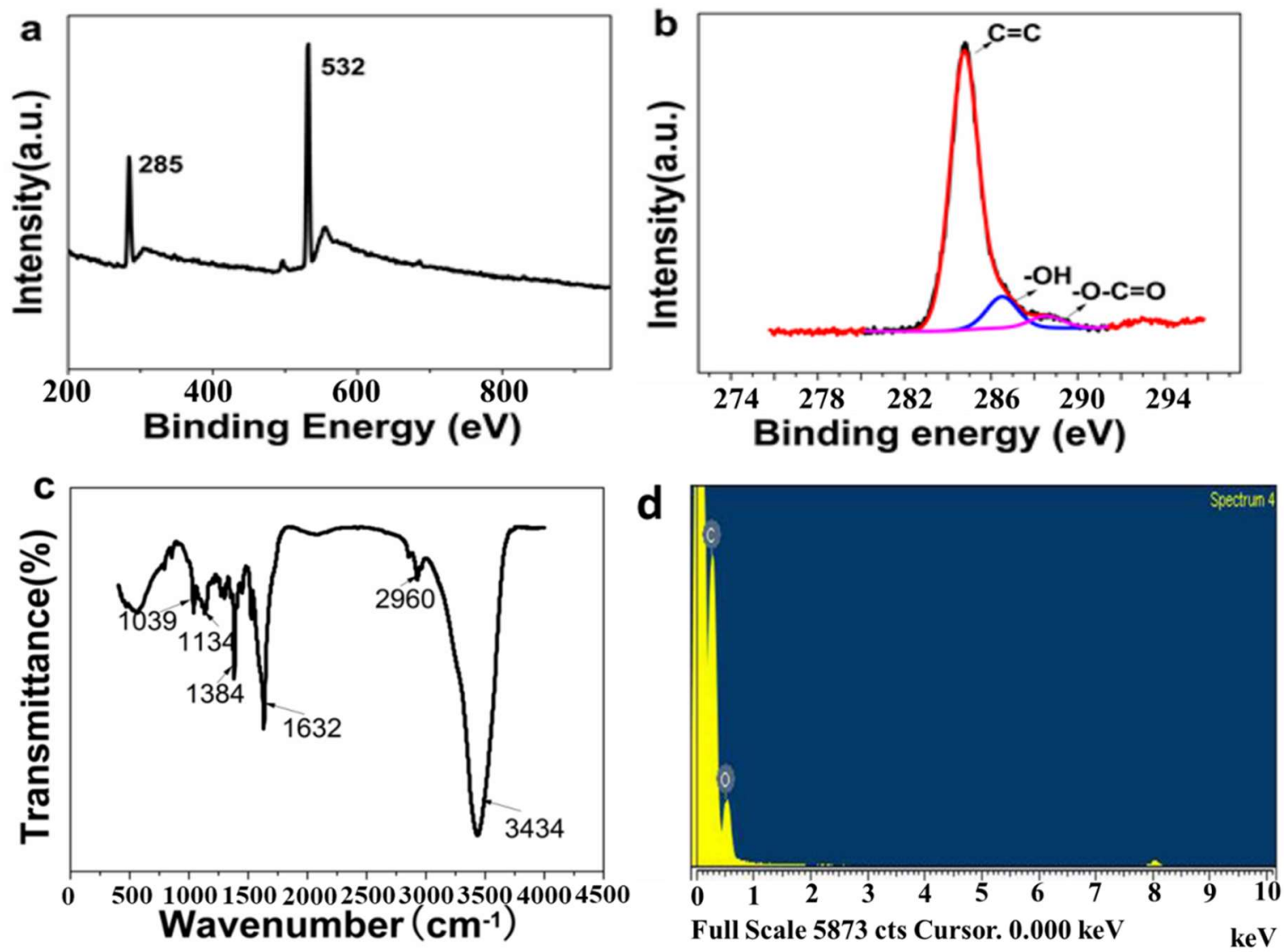

Figure 2. GQDs: (a) X-ray photoelectron spectroscopy (XPS) survey spectrum; (b) high-resolution XPS C1s spectrum; (c) Fourier transform infrared (FTIR) spectrum; (d) Energy disperse spectroscopy (EDS) spectrum. 
In summary, the HOPG was ablated with a pulsed laser along the orientation parallel to the graphite layers in order to fabricate single- or few-layer GQDs with high purity and without chemical additives, residues and stabilizers. These impurities existed in the conventionally chemically synthesized GQDs, and their absence was significant for SERS measurements. On the other hand, although some vibrations related to the conjugate structure of $\mathrm{C}=\mathrm{C}$ and electron-donating substituent groups $(\mathrm{C}-\mathrm{OH}, \mathrm{C}=\mathrm{O}, \mathrm{C}-\mathrm{O}-\mathrm{C})$ were observed, more significant information about the microstructures of the GQD domains, such as in-plane and edge defects, and other defects were not obtained.

In the normal Raman spectrum of the GQDs (curve a of Figure 3a), the G band at $1588 \mathrm{~cm}^{-1}$ and the $\mathrm{D}$ band at $1350 \mathrm{~cm}^{-1}$ were observed [26]. More Raman peaks corresponding to the detailed structures of the GQDs that might be significant for their photoluminescence were not obtained. Curve $b$ of Figure 3a shows the SERS spectrum of GQDs on silver nanoparticles (Ag@GQDs). However, no extra peaks appeared, although the intensities of the D and G bands were enhanced. To further enhance the Raman signals of the GQDs, an electrochemical environment was introduced to tightly combine the Ag@GQD sample and silver electrode. Figure 3b shows the SERS spectra of GQDs on a silver electrode in an aqueous electrochemical environment with varying potentials from $0 \mathrm{~V}$ to -1.2 V. Many new peaks appeared and gradually increased in intensity with potential, indicating that nanosized GQDs with enormous edge defects were adsorbed on the Ag electrode, enhancing the SERS signals of the GQDs.
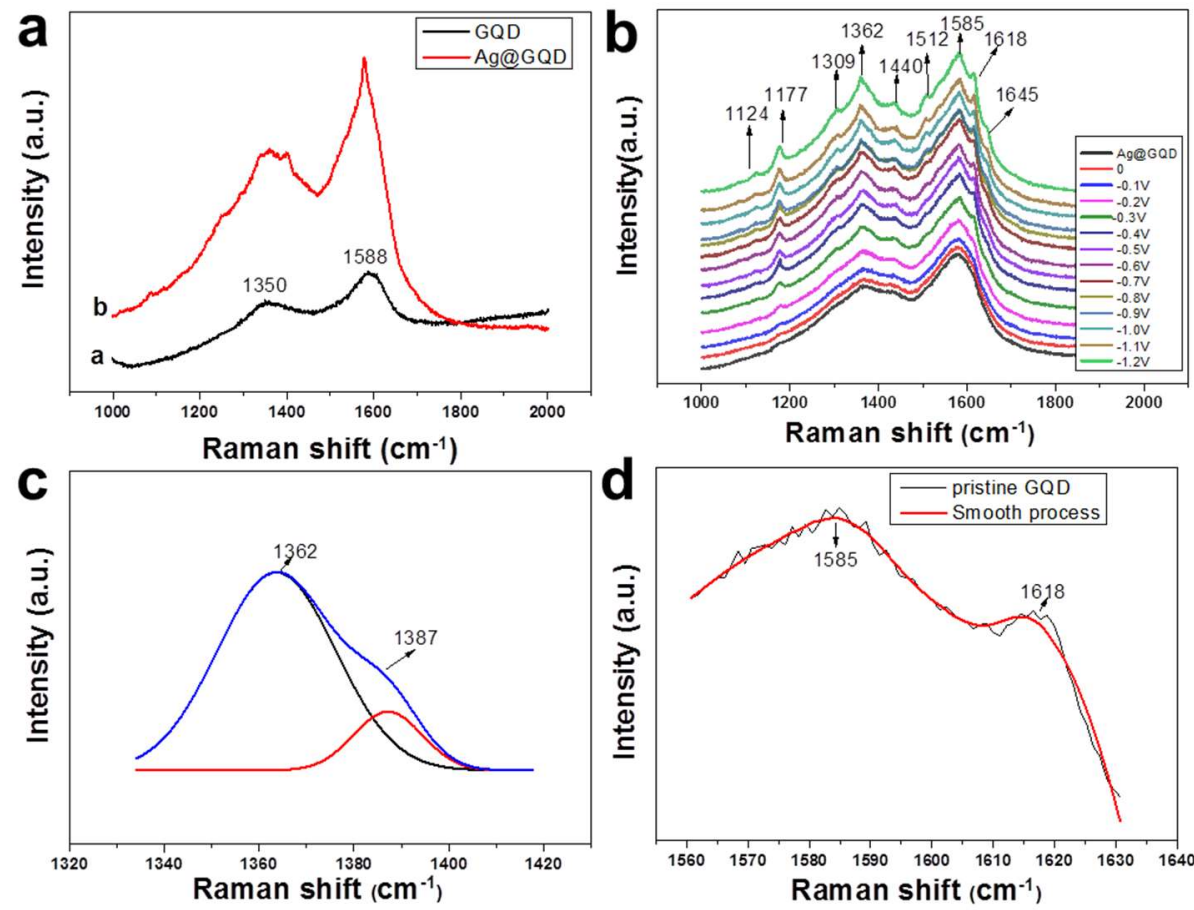

Figure 3. (a) Curve a: Raman spectrum of GQDs on silicon, and curve b: Surface-enhanced Raman spectroscopy (SERS) spectrum of GQDs on silver nanoparticles (Ag@GQDs); (b) SERS spectra of Ag@GQDs coated on a Ag electrode with changing potentials from $0.0 \mathrm{~V}$ to $-1.2 \mathrm{~V}$; (c) The D band in SERS at $-1.2 \mathrm{~V}$ (blue) and two fittings (black and red); and (d) the G band in SERS at $-1.2 \mathrm{~V}$. The excitation wavelength was $532 \mathrm{~nm}$.

The normal Raman intensity ratio of the D band to the $G$ band was 0.77 (Figure 3a), which was similar to that of other high-quality GQDs [19]. This suggests that during the laser ablation process, more defects were formed on the GQDs. The SERS intensity ratio of the D band to the G band increased with decreasing potential and reached 0.96 at $-1.2 \mathrm{~V}$ (Figure 3b). Nanosized GQDs had enough edge defects that the numerical ratio of edge defects to in-plane regular hexagons was much higher than that for large graphene sheets, leading to an increase in the relative intensity of the D band. Some defects 
on the edges of GQD domains can be converted to new defect structures during chemical passivation by electron-donating substituents to form the functional groups, which increase the variety of defects. These functional groups facilitate the chemisorption of GQDs on the Ag electrode and implement charge transfer between GQDs and the Ag surface more readily than the graphitic edge defects of GQD domains. On the other hand, chemical passivation can be strengthened with a decrease in potential, which further increases the charge transfer effect. As a result, the relative intensity of the $D$ band compared to the $G$ band was prominently enhanced. Furthermore, considering that GQDs have various edge defects with complicated structures, the D band in SERS was broadened. Additionally, the calculation also showed that the $\mathrm{D}$ band of graphene could be broadened with a decrease in size because of the quantum confinement effect $[49,50]$.

It is noted that with varying potentials from $0.0 \mathrm{~V}$ to $-1.2 \mathrm{~V}$, the $\mathrm{D}$ band was split into a peak and a shoulder (Figure $3 \mathrm{~b}$ ). Lorentzian fitting provides a reasonable approximation of the measured $\mathrm{D}$ band, indicating that the D band consisted of two peaks at 1362 and $1387 \mathrm{~cm}^{-1}$ (Figure 3c). According to the double resonance theory of graphene, the $\mathrm{D}$ band should be composed of two frequencies. The lower frequency is derived from the defects on the edges, and the higher frequency originates from disorders on the basal planes $[49,50]$. Notably, the intensity of the lower frequency peak of the D band is stronger than that of the higher frequency peak, in contrast to the case of large graphene sheets, in which the lower frequency peak is weaker than the higher frequency peak [51]. GQDs have enormous edge defects. Some of them are chemically passivated by electron-donating substituents, forming functional groups that are more easily chemically adsorbed on Ag electrodes and cause charge transfer between the Ag electrode surface and adsorbed GQDs. This results in the further SERS enhancement of the lower frequency peak. On the other hand, the higher frequency peak, which is related to disorders such as ripple curvature or defects such as carrier doping on the basal planes [39,51], emerges out of the $\mathrm{D}$ band as a weak shoulder. Compared to the ripple disorders on the basal planes of large graphene sheets [51], nanosized GQD domains are nearly flat, reducing the fluctuation in curvature. However, the peak at $1309 \mathrm{~cm}^{-1}$ that is usually ascribed to sp $\mathrm{p}^{3}$ hybridization suggests that some graphitic hexagons on the basal planes of GQD domains or armchairs and zigzags on the edges are damaged and converted into defects such as $\mathrm{sp}^{3}$ structures during laser ablation and probably induce some degree of curvature disorder [52]. Consequently, the lower frequency peak in the D band is much stronger than the higher frequency peak. This double resonance effect, as a typical feature of graphene, also implies that the basal planes of GQD domains retain the quasi-2D sp ${ }^{2}$ hexagonal structure, although enormous defects exist after laser ablation.

Furthermore, the D band shows a blueshift to $1362 \mathrm{~cm}^{-1}$ in SERS compared to its position in normal Raman spectra of graphite, large graphene sheets and GQDs [51,53]. The structures of the edge defects of GQD domains could be redistributed according to the minimum energy principle, leading to a change in the Fermi energy level of the GQDs. The potential applied to the Ag electrode also changed the Fermi energy of the Ag surface. Additionally, the strong quantum confinement effect of GQDs and the charge transfer effect between GQDs and the Ag electrode can tune the lowest unoccupied molecular orbital (LUMO) energy level of GQDs, also leading to a shift in the D band.

For the same reasons the $G$ band also split into two peaks at 1585 and $1618 \mathrm{~cm}^{-1}$ (Figure 3d), which can be attributed to the iTO and LO phonon modes (E2g symmetry), respectively, at the Brillouin zone center [54,55]. In a normal Raman spectrum of large graphene sheets, the $G$ band only has a single G Lorentzian peak because of the energy degeneracy of these two optical phonon modes at the $\Gamma$ point. However, in the SERS of the GQDs, splitting the G band can be induced by the adsorption of GQDs on the Ag electrode, which leads to symmetry breaking.

The mode at $1510 \mathrm{~cm}^{-1}$ was ascribed to the iTO phonon near $1 / 4 \Gamma \mathrm{K}$, and the mode at $1650 \mathrm{~cm}^{-1}$ was assigned to the LO phonon near $\Gamma K / 4,[55,56]$ which are typical features of graphene, further indicating that the GQD domains not only have disorders and defects such as $\mathrm{sp}^{3}$ hybridization on the basal planes, but also basically retain the quasi-2D sp ${ }^{2}$ hexagonal structure. Both new peaks appearing 
at 1124 and $1177 \mathrm{~cm}^{-1}$ were assigned to the vibration of functional groups of $\mathrm{C}-\mathrm{O}$, and the peak located at $1440 \mathrm{~cm}^{-1}$ was attributed to the $\mathrm{C}=\mathrm{C}$ vibration, characterizing the passivated functional groups.

In summary, GQDs can be effectively characterized via SERS. The typical features of nanosized GQDs, such as the crystallinity of $\mathrm{sp}^{2}$ hexagons, the quantum confinement effect, various edge defects, $\mathrm{sp}^{3}$-like defects and disorders on the basal planes of GQDs, and passivated structures on the periphery and surface of GQDs, can be presented by the abundant SERS signals of the GQDs. These results are significant for clearly understanding the PL mechanism in GQDs.

During the synthesis process of GQDs, a number of minute bubbles were produced on the surface of the HOPG as the GQDs were formed by ablating and cutting HOPG with a pulsed laser beam along the orientation parallel to the graphite layers. It is proposed that with laser irradiation, the surface of the HOPG absorbed enough energy over a short time period to increase the temperature in the micro-area. As a result, the graphitic clusters on the laser-ablated micro-area broke out of the HOPG surface. By further overcoming Van der Waals forces, the high-energy graphitic clusters cracked into a single-layer or few-layer nanoscale GQD domains. Simultaneously, water molecules were decomposed to passivate the edges and basal planes of the GQD domains to form the electron-donating substituent groups. GQDs can be stably suspended in water because of the hydrophilicity of the photoinduced functional groups.

\section{Conclusions}

To study the SERS of GQDs, pure GQDs without chemical additives, residues and stabilizers that usually exist in conventionally chemically synthesized GQDs were fabricated by ablating HOPG with a pulsed laser beam along the orientation parallel to the graphite layers in deionized water. To further enhance the SERS signals of GQDs, Ag@GQDs were prepared and adsorbed onto an Ag electrode in an electrochemical environment so that the Ag@GQDs would strongly interact with the Ag electrode at the applied potentials. Consequently, SERS spectra of GQDs with abundant vibrational peaks were obtained. GQDs have various edge defects, including enormous original edge defects and new converted edge defects due to chemical passivation that can be chemisorbed on the Ag electrode to implement charge transfer between the Ag surface and GQDs with potential. This process led to an increase in the intensity of the $D$ band relative to the $G$ band and the broadening and blueshift of the $D$ band. It is noted that D band split into two peaks at 1362 and $1387 \mathrm{~cm}^{-1}$, which can be attributed to the double resonance effect of graphene. Due to the enormous edge defects in GQDs, the lower frequency peak related to edge defects was stronger than the higher frequency peak derived from disorders such as $\mathrm{sp}^{3}$ structures and the fluctuation of the curvature on the basal planes. This is in contrast to the case of large graphene sheets, in which the lower frequency peak is weaker than the higher frequency peak because the numerical ratio of edge defects to in-plane regular hexagons is much lower than that of GQDs. The splitting of the G band and other phonon modes further demonstrate that GQD domains not only have disorders and defects on the basal planes but also basically retain their quasi-2D $\mathrm{sp}^{2}$ hexagonal structure. Some SERS peaks assigned to the vibrations of functional groups were also observed. This work indicates that GQDs can be effectively characterized by SERS. More details of the structures of GQDs that have not been directly observed with other analytical methods are shown in the SERS spectra. The typical features of GQDs-such as the crystallinity of $\mathrm{sp}^{2}$ hexagons, the quantum confinement effect, various defects on the edges, $\mathrm{sp}^{3}$-like defects and disorders on the basal planes, and passivated structures on the periphery and surface of GQDs-were presented by the abundant SERS signals and were significant for clearly understanding the PL mechanism in GQDs. In the future, these microstructures that are characterized by SERS could be well correlated with the GQD PL effect by theoretical simulation of the GQD PL mechanism via the GQD microstructures.

Author Contributions: J.W. and Y.F. conceived and designed the experiments; J.W. performed the experiments; P.W. and F.W. analyzed the data; J.W. and Y.F. wrote the paper.

Acknowledgments: This work was supported by the Natural Science Foundation of China (Grant No. 21473115), and the National High Technology Research and Development Program of China (Grant No.2012AA030303). 
Conflicts of Interest: The authors declare no conflict of interest.

\section{References}

1. Sun, Z.; Chang, H. Graphene and graphene-like two-dimensional materials in photodetection: Mechanisms and methodology. ACS Nano 2014, 8, 4133-4156. [CrossRef] [PubMed]

2. Tayyebi, A.; Akhavan, O.; Lee, B.-K.; Outokesh, M. Supercritical water in top-down formation of tunable-sized graphene quantum dots applicable in effective photothermal treatments of tissues. Carbon 2018, 130, 267-272. [CrossRef]

3. Bian, S.; Shen, C.; Qian, Y.; Liu, J.; Xi, F.; Dong, X. Facile synthesis of sulfur-doped graphene quantum dots as fluorescent sensing probes for $\mathrm{Ag}^{+}$ions detection. Sens. Actuators B 2017, 242, 231-237. [CrossRef]

4. Wang, H.; Revia, R.; Wang, K.; Kant, R.J.; Mu, Q.; Gai, Z.; Hong, K.; Zhang, M. Paramagnetic Properties of Metal-Free Boron-Doped Graphene Quantum Dots and Their Application for Safe Magnetic Resonance Imaging. Adv. Mater. 2017, 29, 1605416. [CrossRef] [PubMed]

5. Luo, Z.; Qi, G.; Chen, K.; Zou, M.; Yuwen, L.; Zhang, X.; Huang, W.; Wang, L. Microwave-Assisted Preparation of White Fluorescent Graphene Quantum Dots as a Novel Phosphor for Enhanced White-Light-Emitting Diodes. Adv. Funct. Mater. 2016, 26, 2739-2744. [CrossRef]

6. Wang, Z.; Yuan, F.; Li, X.; Li, Y.; Zhong, H.; Fan, L.; Yang, S. 53\% Efficient Red Emissive Carbon Quantum Dots for High Color Rendering and Stable Warm White-Light-Emitting Diodes. Adv. Mater. 2017, 29, 1702910. [CrossRef] [PubMed]

7. Kim, D.H.; Kim, T.W. Ultrahigh-luminosity white-light-emitting devices based on edge functionalized graphene quantum dots. Nano Energy 2018, 51, 199-205. [CrossRef]

8. Liu, R.; Yang, R.; Qu, C.; Mao, H.; Hu, Y.; Li, J.; Qu, L. Synthesis of glycine-functionalized graphene quantum dots as highly sensitive and selective fluorescent sensor of ascorbic acid in human serum. Sens. Actuators $B$ 2017, 241, 644-651. [CrossRef]

9. Li, N.; Than, A.; Sun, C.; Tian, J.; Chen, J.; Pu, K.; Dong, X.; Chen, P. Monitoring dynamic cellular redox homeostasis using fluorescence-switchable graphene quantum dots. ACS Nano 2016, 10, 11475-11482. [CrossRef] [PubMed]

10. Iannazzo, D.; Ziccarelli, I.; Pistone, A. Graphene quantum dots: Multifunctional nanoplatforms for anticancer therapy. J. Mater. Chem. B 2017, 5, 6471-6489. [CrossRef]

11. Li, Y.; Zhang, W.; Zhang, L.; Li, J.; Su, Z.; Wei, G. Sequence-Designed Peptide Nanofibers Bridged Conjugation of Graphene Quantum Dots with Graphene Oxide for High Performance Electrochemical Hydrogen Peroxide Biosensor. Adv. Mater. Interfaces 2017, 4, 1600895. [CrossRef]

12. Jeon, S.J.; Kang, T.W.; Ju, J.M.; Kim, M.J.; Park, J.H.; Raza, F.; Han, J.; Lee, H.R.; Kim, J.H. Modulating the photocatalytic activity of graphene quantum dots via atomic tailoring for highly enhanced photocatalysis under visible light. Adv. Funct. Mater. 2016, 26, 8211-8219. [CrossRef]

13. Gupta, S.; Smith, T.; Banaszak, A.; Boeckl, J. Graphene Quantum Dots Electrochemistry and Sensitive Electrocatalytic Glucose Sensor Development. Nanomaterials 2017, 7, 301. [CrossRef] [PubMed]

14. Kim, S.; Hwang, S.W.; Kim, M.-K.; Shin, D.Y.; Shin, D.H.; Kim, C.O.; Yang, S.B.; Park, J.H.; Hwang, E.; Choi, S.-H. Anomalous behaviors of visible luminescence from graphene quantum dots: Interplay between size and shape. ACS Nano 2012, 6, 8203-8208.

15. Yan, Y.; Chen, J.; Li, N.; Tian, J.; Li, K.; Jiang, J.; Liu, J.; Tian, Q.; Chen, P. Systematic Bandgap Engineering of Graphene Quantum Dots and Applications for Photocatalytic Water Splitting and $\mathrm{CO}_{2}$ Reduction. ACS Nano 2018, 12, 3523-3532. [CrossRef] [PubMed]

16. Zheng, X.T.; Ananthanarayanan, A.; Luo, K.Q.; Chen, P. Glowing graphene quantum dots and carbon dots: Properties, syntheses, and biological applications. Small 2015, 11, 1620-1636. [CrossRef] [PubMed]

17. Tang, L.; Ji, R.; Cao, X.; Lin, J.; Jiang, H.; Li, X.; Teng, K.S.; Luk, C.M.; Zeng, S.; Hao, J. Deep ultraviolet photoluminescence of water-soluble self-passivated graphene quantum dots. ACS Nano 2012, 6, 5102-5110. [CrossRef] [PubMed]

18. Wang, Q.; Zheng, H.; Long, Y.; Zhang, L.; Gao, M.; Bai, W. Microwave-hydrothermal synthesis of fluorescent carbon dots from graphite oxide. Carbon 2011, 49, 3134-3140. [CrossRef]

19. Pan, D.; Zhang, J.; Li, Z.; Wu, M. Hydrothermal route for cutting graphene sheets into blue-luminescent graphene quantum dots. Adv. Mater. 2010, 22, 734-738. [CrossRef] [PubMed] 
20. Rozhkov, A.; Giavaras, G.; Bliokh, Y.P.; Freilikher, V.; Nori, F. Electronic properties of mesoscopic graphene structures: Charge confinement and control of spin and charge transport. Phys. Rep. 2011, 503, 77-114. [CrossRef]

21. Zhu, S.; Song, Y.; Wang, J.; Wan, H.; Zhang, Y.; Ning, Y.; Yang, B. Photoluminescence mechanism in graphene quantum dots: Quantum confinement effect and surface/edge state. Nano Today 2017, 13, 10-14. [CrossRef]

22. Gao, T.; Wang, X.; Yang, L.-Y.; He, H.; Ba, X.-X.; Zhao, J.; Jiang, F.-L.; Liu, Y. Red, Yellow, and Blue Luminescence by Graphene Quantum Dots: Syntheses, Mechanism, and Cellular Imaging. ACS Appl. Mater. Interfaces 2017, 9, 24846-24856. [CrossRef] [PubMed]

23. Peng, J.; Gao, W.; Gupta, B.K.; Liu, Z.; Romero-Aburto, R.; Ge, L.; Song, L.; Alemany, L.B.; Zhan, X.; Gao, G. Graphene quantum dots derived from carbon fibers. Nano lett. 2012, 12, 844-849. [CrossRef] [PubMed]

24. Sun, Y.-P.; Zhou, B.; Lin, Y.; Wang, W.; Fernando, K.S.; Pathak, P.; Meziani, M.J.; Harruff, B.A.; Wang, X.; Wang, H. Quantum-sized carbon dots for bright and colorful photoluminescence. J. Am. Chem. Soc. 2006, 128, 7756-7757. [CrossRef] [PubMed]

25. Jin, S.H.; Kim, D.H.; Jun, G.H.; Hong, S.H.; Jeon, S. Tuning the photoluminescence of graphene quantum dots through the charge transfer effect of functional groups. ACS Nano 2013, 7, 1239-1245. [CrossRef] [PubMed]

26. Zhu, S.; Zhang, J.; Qiao, C.; Tang, S.; Li, Y.; Yuan, W.; Li, B.; Tian, L.; Liu, F.; Hu, R. Strongly green-photoluminescent graphene quantum dots for bioimaging applications. Chem. Commun. 2011, 47, 6858-6860. [CrossRef] [PubMed]

27. Chen, W.; Lv, G.; Hu, W.; Li, D.; Chen, S.; Dai, Z. Synthesis and applications of graphene quantum dots: A review. Nanotechnol. Rev. 2018, 7, 157. [CrossRef]

28. Permatasari, F.A.; Aimon, A.H.; Iskandar, F.; Ogi, T.; Okuyama, K. Role of C-N configurations in the photoluminescence of graphene quantum dots synthesized by a hydrothermal route. Sci. Rep. 2016, 6, 21042. [CrossRef] [PubMed]

29. Diao, S.; Zhang, X.; Shao, Z.; Ding, K.; Jie, J.; Zhang, X. 12.35\% efficient graphene quantum dots/silicon heterojunction solar cells using graphene transparent electrode. Nano Energy 2017, 31, 359-366. [CrossRef]

30. Gupta, S.; Walden, J.; Banaszak, A.; Carrizosa, S.B. Facile Synthesis of Water-Soluble Graphene Quantum Dots/Graphene for Efficient Photodetector. MRS Adv. 2018, 3, 817-824. [CrossRef]

31. Shen, J.; Zhu, Y.; Chen, C.; Yang, X.; Li, C. Facile preparation and upconversion luminescence of graphene quantum dots. Chem. Commun. 2011, 47, 2580-2582. [CrossRef] [PubMed]

32. Zhu, H.; Wang, X.; Li, Y.; Wang, Z.; Yang, F.; Yang, X. Microwave synthesis of fluorescent carbon nanoparticles with electrochemiluminescence properties. Chem. Commun. 2009, 34, 5118-5120. [CrossRef] [PubMed]

33. Vázquez-Nakagawa, M.; Rodríguez-Pérez, L.; Herranz, M.; Martín, N. Chirality transfer from graphene quantum dots. Chem. Commun. 2016, 52, 665-668. [CrossRef] [PubMed]

34. Huang, H.; Yang, S.; Li, Q.; Yang, Y.; Wang, G.; You, X.; Mao, B.; Wang, H.; Ma, Y.; He, P. Electrochemical Cutting in Weak Aqueous Electrolytes: The Strategy for Efficient and Controllable Preparation of Graphene Quantum Dots. Langmuir 2017, 34, 250-258. [CrossRef] [PubMed]

35. Yan, Y.; Li, H.; Wang, Q.; Mao, H.; Kun, W. Controllable ionic liquid-assisted electrochemical exfoliation of carbon fibers for the green and large-scale preparation of functionalized graphene quantum dots endowed with multicolor emission and size tunability. J. Mater. Chem. C 2017, 5, 6092-6100. [CrossRef]

36. Shen, J.; Zhu, Y.; Yang, X.; Zong, J.; Zhang, J.; Li, C. One-pot hydrothermal synthesis of graphene quantum dots surface-passivated by polyethylene glycol and their photoelectric conversion under near-infrared light. New J. Chem. 2012, 36, 97-101. [CrossRef]

37. Pan, D.; Guo, L.; Zhang, J.; Xi, C.; Xue, Q.; Huang, H.; Li, J.; Zhang, Z.; Yu, W.; Chen, Z. Cutting sp 2 clusters in graphene sheets into colloidal graphene quantum dots with strong green fluorescence. J. Mater. Chem. 2012, 22, 3314-3318. [CrossRef]

38. Park, J.; Reina, A.; Saito, R.; Kong, J.; Dresselhaus, G.; Dresselhaus, M. G' band Raman spectra of single, double and triple layer graphene. Carbon 2009, 47, 1303-1310. [CrossRef]

39. Merlen, A.; Buijnsters, J.G.; Pardanaud, C. A Guide to and Review of the Use of Multiwavelength Raman Spectroscopy for Characterizing Defective Aromatic Carbon Solids: From Graphene to Amorphous Carbons. Coatings 2017, 7, 153. [CrossRef]

40. Ferrari, A.C.; Basko, D.M. Raman spectroscopy as a versatile tool for studying the properties of graphene. Nat. Nanotechnol. 2013, 8, 235. [CrossRef] [PubMed] 
41. Budde, H.; Coca-López, N.; Shi, X.; Ciesielski, R.; Lombardo, A.; Yoon, D.; Ferrari, A.C.; Hartschuh, A. Raman Radiation Patterns of Graphene. ACS Nano 2016, 10, 1756-1763. [CrossRef] [PubMed]

42. Sun, Z.; Hasan, T.; Torrisi, F.; Popa, D.; Privitera, G.; Wang, F.; Bonaccorso, F.; Basko, D.M.; Ferrari, A.C. Graphene Mode-Locked Ultrafast Laser. ACS Nano 2010, 4, 803-810. [CrossRef] [PubMed]

43. Mohiuddin, T.M.G.; Lombardo, A.; Nair, R.R.; Bonetti, A.; Savini, G.; Jalil, R.; Bonini, N.; Basko, D.M.; Galiotis, C.; Marzari, N.; et al. Uniaxial strain in graphene by Raman spectroscopy: G peak splitting, Grüneisen parameters, and sample orientation. Phys. Rev. B 2009, 79, 205433. [CrossRef]

44. Kneipp, K.; Kneipp, H.; Itzkan, I.; Dasari, R.R.; Feld, M.S. Ultrasensitive chemical analysis by Raman spectroscopy. Chem. Rev. 1999, 99, 2957-2976. [CrossRef] [PubMed]

45. Gupta, S.; Banaszak, A.; Smith, T.; Dimakis, N. Molecular sensitivity of metal nanoparticles decorated graphene-family nanomaterials as surface-enhanced Raman scattering (SERS) platforms. J. Raman Spectrosc. 2018, 49, 438-451. [CrossRef]

46. Wu, D.-Y.; Li, J.-F.; Ren, B.; Tian, Z.-Q. Electrochemical surface-enhanced Raman spectroscopy of nanostructures. Chem. Soc. Rev. 2008, 37, 1025-1041. [CrossRef] [PubMed]

47. Hu, S.-L.; Niu, K.-Y.; Sun, J.; Yang, J.; Zhao, N.-Q.; Du, X.-W. One-step synthesis of fluorescent carbon nanoparticles by laser irradiation. J. Mater. Chem. 2009, 19, 484-488. [CrossRef]

48. Cui, L.; Xu, Y.; Liu, B.; Yang, W.; Song, Z.; Liu, J. Well-controlled preparation of evenly distributed nanoporous HOPG surface via diazonium salt assisted electrochemical etching process. Carbon 2016, 102, 419-425. [CrossRef]

49. Saito, R.; Jorio, A.; Souza Filho, A.; Dresselhaus, G.; Dresselhaus, M.; Pimenta, M. Probing phonon dispersion relations of graphite by double resonance Raman scattering. Phys. Rev. Lett. 2001, 88, 027401. [CrossRef] [PubMed]

50. Nemanich, R.J.; Solin, S. First- and second-order Raman scattering from finite-size crystals of graphite. Phys. Rev. B 1979, 20, 392. [CrossRef]

51. Li, L.; An, B.; Lahiri, A.; Wang, P.; Fang, Y. Doublet of D and 2D bands in graphene deposited with Ag nanoparticles by surface enhanced Raman spectroscopy. Carbon 2013, 65, 359-364. [CrossRef]

52. Zhang, Y.; Shang, J.; Fu, W.; Zeng, L.; Tang, T.; Cai, Y. A sp ${ }^{2}+\mathrm{sp}^{3}$ hybridized carbon allotrope transformed from AB stacking graphyne and THD-graphene. AIP Adv. 2018, 8, 015028. [CrossRef]

53. Kaniyoor, A.; Ramaprabhu, S. A Raman spectroscopic investigation of graphite oxide derived graphene. AIP Adv. 2012, 2, 032183. [CrossRef]

54. Dong, X.; Shi, Y.; Zhao, Y.; Chen, D.; Ye, J.; Yao, Y.; Gao, F.; Ni, Z.; Yu, T.; Shen, Z. Symmetry breaking of graphene monolayers by molecular decoration. Phys. Rev. Lett. 2009, 102, 135501. [CrossRef] [PubMed]

55. Kawashima, Y.; Katagiri, G. Fundamentals, overtones, and combinations in the Raman spectrum of graphite. Phys. Rev. B 1995, 52, 10053. [CrossRef]

56. Thomsen, C.; Reich, S. Double resonant Raman scattering in graphite. Phys. Rev. Lett. 2000, 85, 5214. [CrossRef] [PubMed]

(C) 2018 by the authors. Licensee MDPI, Basel, Switzerland. This article is an open access article distributed under the terms and conditions of the Creative Commons Attribution (CC BY) license (http:// creativecommons.org/licenses/by/4.0/). 